\title{
Maximal Characterization of Locally Summable Functions
}

\author{
F. Andreano and R. Grande
}

Abstract. We prove a characterization of locally summable functions with bounded Stepanoff norm through the maximal function

$$
M_{\phi} f(x)=\sup _{t>0}\left|\left(f * \phi_{t}\right)(x)\right|
$$

where $\phi$ is a suitable function in the class of Schwartz.

Keywords. Stepanoff bounded functions, maximal function

Mathematics Subject Classification (2000). 42A75, 42A45

\section{Introduction}

Given $\phi$ in the Schwartz class $\mathcal{S}$, the maximal function $M_{\phi}$ of a distribution $f$ is

$$
M_{\phi} f(x)=\sup _{t>0}\left|\left(f * \phi_{t}\right)(x)\right|,
$$

where $\phi_{t}(x)=\frac{1}{t} \phi\left(\frac{x}{t}\right)$. The following maximal characterization for $L^{p}(\mathbb{R})$ is well known (cfr. [5]).

Theorem 1.1. Let $1<p \leq+\infty$. If $f$ is a distribution, then:

$$
f \in L^{p}(\mathbb{R}) \Longleftrightarrow \exists \phi \in \mathcal{S}, \text { with } \int \phi d x \neq 0, \text { so that } M_{\phi} f \in L^{p}(\mathbb{R}) .
$$

It is interesting to consider maximal characterizations of spaces of functions which are only locally summable, that is, "big" at infinity. This problem has been suggested by A. Pankov in a seminar given at the Department of Metodi e Modelli Matematici per le Scienze Applicate at Università di Roma "La Sapienza".

First we need to introduce an appropriate Banach space structure on locally $L^{p}$ functions. Let us define the spaces $\operatorname{BS}^{p}(\mathbb{R})$ of Stepanoff bounded functions.

F. Andreano, R. Grande: Università di Roma "La Sapienza", Dipartimento di Metodi e Modelli Matematici per le Scienze Applicate, Via Scarpa 16, 00161 Rome, Italy; andreano@dmmm.uniroma1.it,grande@dmmm.uniroma1.it 
Definition 1.2. Let $1 \leq p<+\infty$. Then $f \in \mathrm{BS}^{p}(\mathbb{R})$ if

1. $f \in L_{l o c}^{p}(\mathbb{R})$;

2. $\sup _{x \in \mathbb{R}} \int_{x}^{x+1}|f(t)|^{p} d t \leq c$, for $c \in \mathbb{R}$.

Note 1.3.

1. $\|f\|_{S^{p}}=\sup _{x \in \mathbb{R}}\left(\int_{x}^{x+1}|f(t)|^{p} d t\right)^{\frac{1}{p}}$ is a norm; such norm is equivalent to the norm

$$
\|f\|_{S_{l}^{p}}=\sup _{x \in \mathbb{R}}\left(\frac{1}{l} \int_{x}^{x+l}|f(t)|^{p} d t\right)^{\frac{1}{p}}
$$

where $l \in \mathbb{R}_{+}($cfr. [1]).

2. The space $\operatorname{BS}^{p}(\mathbb{R})$ contains the space of Stepanoff almost-periodic functions $S^{p}(\mathbb{R})$, i.e., the space of functions that can be approximated by trigonometric polynomials in the Stepanoff norm $\|\cdot\|_{S^{p}}$ defined above (cfr. [1] and [4]).

We prove the following maximal characterization of $\mathrm{BS}^{p}(\mathbb{R})$ :

Theorem 1.4. Let $1<p<+\infty$. If $f$ is a distribution, then:

$$
f \in \mathrm{BS}^{p}(\mathbb{R}) \Longleftrightarrow \exists \phi \in \mathcal{S}, \text { with } \int \phi d x \neq 0, \text { so that } M_{\phi} f \in \mathrm{BS}^{p}(\mathbb{R}) .
$$

For this we need to prove an analogue, for $\mathrm{BS}^{p}(\mathbb{R})$, of the Hardy-Littlewood maximal theorem. As already observed, a similar result is known for $L^{p}$. The proof on $L^{p}$, however, does not readly extend to this more general framework, because, in the case of $L^{p}(\mathbb{R})$, one uses the weak compactness of $L^{p}(\mathbb{R})$ to prove the sufficient part of the maximal characterization. The dual space of $\mathrm{BS}^{p}(\mathbb{R})$ is not known and therefore we do not have a weak convergence result in such spaces.

\section{The maximal characterization for $\mathrm{BS}^{p}(\mathbb{R})$}

In this section we prove Theorem 1.4. The necessary part follows from the Hardy-Littlewood maximal theorem for $\mathrm{BS}^{p}(\mathbb{R})$, that we prove separately.

Let $M f$ be the maximal function of $f$, defined by

$$
M f(x)=\sup _{x \in I} \frac{1}{m(I)} \int_{I}|f(t)| d t
$$

where the supremum is taken over all intervals $I$ containing $x$. Here $m$ denotes the Lebesgue measure.

Note 2.1. $M_{\phi} f(x) \leq c M f(x)$, where $c$ is a constant (for the proof of this inequality see [5, Chapter 2, Section 2.1]). 
Let $l>0$ and $M_{\nu, l} f(x)$ be the maximal function of $f$ restricted to $[\nu, \nu+l]$, $\nu \in \mathbb{R}$, i.e., let

$$
M_{\nu, l} f(x)=\sup _{x \in I \subseteq[\nu, \nu+l]} \frac{1}{m(I)} \int_{I}|f(t)| d t,
$$

if $x \in[\nu, \nu+l]$, and $M_{\nu, l} f(x)=0$, if $x \notin[\nu, \nu+l]$.

Furthermore let

$$
m_{f}(\alpha, \nu, l)=m\left(\left\{x \in[\nu, \nu+l]: M_{\nu, l} f(x)>\alpha\right\}\right),
$$

with $\alpha, l \in \mathbb{R}_{+}, \nu \in \mathbb{R}$.

Then we have the following

Lemma 2.2. For any $\nu \in \mathbb{R}$ and $l>0, \alpha>0$, and $f \in \mathrm{BS}^{p}(\mathbb{R}), p>1$,

and

$$
m_{f}(\alpha, \nu, l) \leq \frac{4}{\alpha} \int_{\left\{x \in[\nu, \nu+l]: M_{\nu, l} f(x)>\frac{\alpha}{2}\right\}}|f(t)| d t
$$

$$
\frac{1}{l} \int_{\nu}^{\nu+l}\left|M_{\nu, l} f(t)\right|^{p} d t \leq \frac{2^{p+1} p}{p-1} \frac{1}{l} \int_{\nu}^{\nu+l}|f(t)|^{p} d t .
$$

The proof is analogous to the proof of Theorem 4.3 in [3, Chapter 1].

Theorem 2.3 (Hardy-Littlewood maximal theorem for $\mathrm{BS}^{p}(\mathbb{R})$ ). Let $p>1$, $f \in \mathrm{BS}^{p}(\mathbb{R})$, and let $l>0$. There exists $c>0$ such that

$$
\|M f\|_{S_{l}^{p}} \leq c\|f\|_{S_{l}^{p}}
$$

Proof. Let $\nu \in \mathbb{R}$ and $l>0$. Set

$$
\Pi_{1}=\{I: I \subseteq[\nu-l,(\nu-l)+3 l]\}
$$

and

$$
\Pi_{2}=\{J: J \cap(\mathbb{R} \backslash[\nu-l,(\nu-l)+3 l]) \neq \emptyset\},
$$

where $I, J$ are intervals. Furthermore set

$$
N_{\nu-l, 3 l} f(x)=\sup _{x \in J \in \Pi_{2}} \frac{1}{m(J)} \int_{J}|f(t)| d t .
$$

Then, for all $x \in \mathbb{R}, M f(x)=\max \left\{M_{\nu-l, 3 l} f(x), N_{\nu-l, 3 l} f(x)\right\}$. By Lemma 2.2, it suffices to prove that $N_{\nu-l, 3 l} f(x) \leq c\|f\|_{S_{l}^{p}}$, for $x \in[\nu, \nu+l]$.

Let $J=[a, b] \in \Pi_{2}$. Since $x \in[\nu, \nu+l]$,

$$
a<\nu-l \text { and } b>\nu
$$


or else

$$
\nu-l<a<\nu+l \text { and } b>(\nu-l)+3 l
$$

and hence, in both cases, we have that $l^{\prime}=b-a>l$. We can write

$$
\begin{aligned}
\frac{1}{m(J)} \int_{J}|f(t)| d t & =\frac{1}{b-a} \int_{a}^{b}|f(t)| d t \\
& =\frac{1}{b-a} \int_{a}^{a+(b-a)}|f(t)| d t \\
& =\frac{1}{l^{\prime}} \int_{a}^{a+l^{\prime}}|f(t)| d t .
\end{aligned}
$$

Since $l^{\prime}>l$, we may write $l^{\prime}=n l+\vartheta l$, with $n \in \mathbb{N}$ and $0<\vartheta<1$. Hence

$$
\begin{aligned}
\frac{1}{l^{\prime}} \int_{a}^{a+l^{\prime}}|f(t)| d t & =\frac{1}{n l+\vartheta l} \int_{a}^{a+n l+\vartheta l}|f(t)| d t \\
& <\frac{1}{n l} \int_{a}^{a+(n+1) l}|f(t)| d t \\
& \leq \frac{1}{n l}\left\{\int_{a}^{a+l}|f(t)| d t+\cdots+\int_{a+n l}^{a+(n+1) l}|f(t)| d t\right\} \\
& \leq \frac{n+1}{n} \sup _{a \in \mathbb{R}} \frac{1}{l} \int_{a}^{a+l}|f(t)| d t \\
& \leq 2 \sup _{a \in \mathbb{R}} \frac{1}{l} \int_{a}^{a+l}|f(t)| d t \leq 2\|f\|_{S_{l}^{p}}
\end{aligned}
$$

and therefore $N_{\nu-l, 3 l} f(x) \leq 2\|f\|_{S_{l}^{p}}$, for $x \in[\nu, \nu+l]$, and hence the thesis of the theorem is proved.

In the proof of Theorem 1.4 we use a result due to R. Doss (cfr. [2]). For completeness we state that theorem:

Theorem 2.4. Let $\left\{\sigma_{m}(x)\right\}$ be a sequence of functions summable in every finite interval and verifying the following condition: to every $\epsilon>0$ there corresponds $a \delta>0$ such that, for every set $E$ of diameter less than or equal to 1 and of measure less than or equal to $\delta$,

$$
\int_{E}\left|\sigma_{m}(x)\right| d x \leq \epsilon, \quad \forall m .
$$

Then there exists a summable function $\sigma(x)$ and a subsequence $\left\{\sigma_{m_{k}}\right\}$ such that, for every bounded function $f(x)$ and every finite interval $(a, b)$,

$$
\lim _{k \rightarrow \infty} \int_{a}^{b} f(x) \sigma_{m_{k}}(x) d x=\int_{a}^{b} f(x) \sigma(x) d x .
$$


Proof of Theorem 1.4. We first prove that $M_{\phi} f \in \mathrm{BS}^{p}$, for all $f \in \mathrm{BS}^{p}$. Let $f \in \mathrm{BS}^{p}(\mathbb{R})$. By Theorem 2.3, we have that there exists $c>0$ such that $\|M f\|_{S^{p}} \leq c\|f\|_{S^{p}}$. Let $\phi \in \mathcal{S}$ and $M_{\phi} f(x)=\sup _{t>0}\left|\left(f * \phi_{t}\right)(x)\right|$. Then there exists $c^{\prime}>0$ such that $M_{\phi} f(x) \leq c^{\prime} M f(x)$ (see Note 2.1). Hence

$$
\left\|M_{\phi} f\right\|_{S^{p}} \leq c^{\prime}\|M f\|_{S^{p}} \leq c^{\prime} c\|f\|_{S^{p}}
$$

and $M_{\phi} f \in \mathrm{BS}^{p}(\mathbb{R})$.

Viceversa, suppose that $M_{\phi} f \in \mathrm{BS}^{p}(\mathbb{R})$, with $\phi \in \mathcal{S}$ such that $\int \phi=1$. We want to show that $f \in \mathrm{BS}^{p}$. Let us consider the sequence $\left(f * \phi_{\frac{1}{n}}\right)(x)$. We have that

$$
\left\|f * \phi_{\frac{1}{n}}\right\|_{S^{p}}=\sup _{x \in \mathbb{R}}\left(\int_{x}^{x+1}\left|\left(f * \phi_{\frac{1}{n}}\right)\right|^{p} d t\right)^{\frac{1}{p}} .
$$

Since $M_{\phi} f \in \mathrm{BS}^{p}(\mathbb{R})$,

$$
\sup _{x \in \mathbb{R}} \int_{x}^{x+1}\left|\left(f * \phi_{\frac{1}{n}}\right)\right|^{p} d t \leq \sup _{x \in \mathbb{R}} \int_{x}^{x+1}\left(\sup _{s>0}\left|\left(f * \phi_{s}\right)(t)\right|\right)^{p} d t \leq B^{p}<+\infty,
$$

where $B$ is a constant, and hence $\left\|f * \phi_{\frac{1}{n}}\right\|_{S^{p}} \leq B<+\infty$, i.e., $f * \phi_{\frac{1}{n}}$ is a bounded sequence in $\mathrm{BS}^{p}(\mathbb{R})$.

Set $h_{n}=f * \phi_{\frac{1}{n}}$. We want to show that there exists a subsequence $\left\{h_{n_{j}}\right\}_{j \in \mathbb{N}}$ and a function $f_{o} \in \mathrm{BS}^{p}(\mathbb{R})$ such that for any measurable and bounded function $\varphi$ and for any bounded interval $(a, b) \subset \mathbb{R}$, one has

$$
\lim _{j \rightarrow+\infty} \int_{a}^{b} \varphi(t) h_{n_{j}}(t) d t=\int_{a}^{b} \varphi(t) f_{o}(t) d t
$$

We apply Theorem 2.4 (cfr. [2]) in order to get that there exists a function $f_{o} \in L_{l o c}^{1}(\mathbb{R})$ verifying (1) for any measurable bounded function $\varphi$ and for any bounded interval $(a, b)$ in $\mathbb{R}$. In order to do this, we need to prove that, if $E$ is any measurable set such that $m(E) \rightarrow 0$, then $\int_{E}\left|h_{n}(t)\right| d t \rightarrow 0$ uniformly with respect to $n \in \mathbb{N}$.

Let $E$ be measurable such that $m(E) \rightarrow 0$. The diameter of $E$ is therefore less than 1 and hence $E \subset(x, x+1)$, for $x \in \mathbb{R}$ suitably chosen. Therefore

$$
\begin{aligned}
\int_{E}\left|h_{n}(t)\right| d t & =\int_{x}^{x+1} \chi_{E}(t)\left|h_{n}(t)\right| d t \\
& \leq\left(\int_{x}^{x+1}\left|h_{n}(t)\right|^{p} d t\right)^{\frac{1}{p}}\left(\int_{x}^{x+1} \chi_{E}(t) d t\right)^{\frac{1}{q}} \\
& \leq \sup _{x \in \mathbb{R}}\left(\int_{x}^{x+1}\left|h_{n}(t)\right|^{p} d t\right)^{\frac{1}{p}}[m(E)]^{\frac{1}{q}} \\
& \leq B[m(E)]^{\frac{1}{q}}
\end{aligned}
$$


where $\frac{1}{p}+\frac{1}{q}=1$. Hence $\int_{E}\left|h_{n}(t)\right| d t \rightarrow 0$, if $m(E) \rightarrow 0$, uniformly with respect to $n \in \mathbb{N}$. The hypotheses of Theorem 2.4 are satisfied and therefore (1) holds with $f_{o} \in L_{l o c}^{1}(\mathbb{R})$.

We need to prove that $f_{o} \in \mathrm{BS}^{p}(\mathbb{R})$. To see this, let $\delta \in(0,1)$ and set, for $n \in \mathbb{N}$,

$$
f_{o}^{(\delta)}(x)=\frac{1}{\delta} \int_{x}^{x+\delta} f_{o}(t) d t \quad \text { and } \quad h_{n}^{(\delta)}(x)=\frac{1}{\delta} \int_{x}^{x+\delta} h_{n}(t) d t .
$$

Since the integral function can be derivated a.e., using Lebesgue's theorem we get

$$
\begin{array}{lll}
\lim _{\delta \rightarrow 0} f_{o}^{(\delta)}(x)=f_{o}(x), & x \in \mathbb{R} & \text { a.e. } \\
\lim _{\delta \rightarrow 0} h_{n}^{(\delta)}(x)=h_{n}(x), & x \in \mathbb{R} & \text { a.e.. }
\end{array}
$$

Furthermore, by (1), we get that

$$
\lim _{j \rightarrow+\infty} h_{n_{j}}^{(\delta)}(x)=f_{o}^{(\delta)}(x), \quad \forall x \in \mathbb{R}, \forall \delta \in(0,1) .
$$

Hence, by Fatou's lemma

$$
\int_{x}^{x+1}\left|f_{o}^{(\delta)}(t)\right|^{p} d t \leq \liminf _{j \rightarrow+\infty} \int_{x}^{x+1}\left|h_{n_{j}}^{(\delta)}(t)\right|^{p} d t .
$$

In order to prove that $f_{o} \in \mathrm{BS}^{p}(\mathbb{R})$, we need to show first that $\int_{x}^{x+1}\left|f_{o}^{(\delta)}(t)\right|^{p} d t$ is bounded independently of $x$. To see this, consider

$$
\begin{aligned}
\int_{x}^{x+1}\left|h_{n_{j}}^{(\delta)}(t)\right|^{p} d t & =\int_{x}^{x+1}\left(\frac{1}{\delta}\left|\int_{t}^{t+\delta} h_{n_{j}}(s) d s\right|\right)^{p} d t \\
& \leq \int_{x}^{x+1}\left(\frac{1}{\delta} \int_{t}^{t+\delta}\left|h_{n_{j}}(s)\right| d s\right)^{p} d t .
\end{aligned}
$$

Consider now

$$
\frac{1}{\delta} \int_{t}^{t+\delta}\left|h_{n_{j}}(s)\right| d s \leq \frac{1}{\delta}\left(\int_{t}^{t+\delta}\left|h_{n_{j}}(s)\right|^{p} d s\right)^{\frac{1}{p}} \delta^{\frac{1}{q}}=\left(\frac{1}{\delta} \int_{t}^{t+\delta}\left|h_{n_{j}}(s)\right|^{p} d s\right)^{\frac{1}{p}} .
$$

Hence

$$
\begin{aligned}
\liminf _{j \rightarrow+\infty} \int_{x}^{x+1}\left|h_{n_{j}}^{(\delta)}(t)\right|^{p} d t & \leq \liminf _{j \rightarrow+\infty} \int_{x}^{x+1} \frac{1}{\delta} \int_{t}^{t+\delta}\left|h_{n_{j}}(s)\right|^{p} d s d t \\
& \leq \liminf _{j \rightarrow+\infty}\left(\int_{x}^{x+1+\delta}\left|h_{n_{j}}(s)\right|^{p} d s\right)\left(\frac{1}{\delta} \int_{s-\delta}^{s} d t\right) \\
& =\liminf _{j \rightarrow+\infty} \int_{x}^{x+1+\delta}\left|h_{n_{j}}(s)\right|^{p} d s \\
& \leq \liminf _{j \rightarrow+\infty} \int_{x}^{x+2}\left|h_{n_{j}}(s)\right|^{p} d s \\
& \leq 2 B^{p}<+\infty,
\end{aligned}
$$


and so $\int_{x}^{x+1}\left|f_{o}^{(\delta)}(t)\right|^{p} d t \leq 2 B^{p}$. Applying once more Fatou's lemma, we get

$$
\int_{x}^{x+1}\left|f_{o}(t)\right|^{p} d t \leq \liminf _{\delta \rightarrow 0} \int_{x}^{x+1}\left|f_{o}^{(\delta)}(t)\right|^{p} d t \leq 2 B^{p} .
$$

Hence

$$
\sup _{x \in \mathbb{R}}\left(\int_{x}^{x+1}\left|f_{o}(t)\right|^{p} d t\right)^{\frac{1}{p}} \leq 2^{\frac{1}{p}} B<+\infty
$$

and $f_{o} \in \mathrm{BS}^{p}(\mathbb{R})$.

We have shown that there exists $f_{o} \in \mathrm{BS}^{p}(\mathbb{R})$ such that

$$
\lim _{j \rightarrow+\infty} \int_{a}^{b} \varphi(x) h_{n_{j}}(x) d x=\lim _{j \rightarrow+\infty} \int_{a}^{b} \varphi(x)\left(f * \phi_{\frac{1}{n_{j}}}\right)(x) d x=\int_{a}^{b} \varphi(x) f_{o}(x) d x
$$

for any measurable bounded function $\varphi$ and for any bounded interval $(a, b) \subset \mathbb{R}$.

On the other hand $f * \phi_{\frac{1}{n_{j}}} \rightarrow f$ as $j \rightarrow+\infty$ in the sense of distributions, and so $f=f_{o} \in \mathrm{BS}^{p}(\mathbb{R})$.

\section{References}

[1] Besicovitch, A. S., Almost Periodic Functions. Cambridge: Cambridge University Press 1932.

[2] Doss, R., Some Theorems on Almost-Periodic Functions. Amer. J. Math. 72 (1950), $81-92$.

[3] Garnett, J. B., Bounded Analytic Functions. New York: Academic Press 1981.

[4] Pankov, A. A., Bounded and Almost Periodic Solutions of Nonlinear Operator Differential Equations. Dordrecht: Kluwer 1990.

[5] Stein, E. M., Harmonic Analysis: Real-Variable Methods, Orthogonality, and Oscillatory Integrals. Princeton (NJ): Princeton University Press 1993.

Received June 1, 2005 